\title{
Use of magnesium sulfate to manage pre-eclampsia and eclampsia in Nigeria: Overcoming the odds
}

\author{
B. A. Ekele \\ Department of Obstetrics and Gynecology, Usmanu Danfodiyo University, Sokoto, Nigeria
}

Page 173

Correspondenceto: Prof. B. A. Ekele, Department of Obstetrics and Gynecology, Usmanu Danfodiyo University, Sokoto, Nigeria. E-mail: bissekele@ yahoo.com

DOI: 10.4103/1596-3519.56231 PMID: 19805934

Professor B A Ekele is a member of the International Society for the Study of Hypertension in Pregnancy (ISSHP) and was a centre coordinator for the Magpie Trial.

Pre-eclampsia and eclampsia are hypertensive disorders of pregnancy that cause significant morbidity and mortality in the fetus and mother both in the developed and developing countries. ${ }^{[1]}$ In Nigeria, they are listed as one of the top three causes of maternal mortality. ${ }^{[2-8]}$ Ultimately, the only "cure" for pre-eclampsia and eclampsia is delivery of the baby. However, maternal and perinatal deaths are significantly reduced with appropriate treatment.

The use of anticonvulsants to manage pre-eclampsia and eclampsia has undergone a major change since the publications of two, large, multi-centered, randomized controlled studies. The first was the collaborative eclampsia trial of 1995 that produced compelling evidence in support of magnesium sulfate as the anticonvulsant of first choice both to control seizures and to prevent recurrence in eclampsia. ${ }^{[9]}$ While the Magpie trial was on patients with pre-eclampsia and the conclusion was that magnesium sulfate reduces the risk of eclampsia and maternal deaths. ${ }^{[10]}$ It is instructive to note that four centers in Nigeria (Ibadan, Port Harcourt, Sagamu and Sokoto) participated actively in the Magpie study. There was also a follow-up study on the babies whose mothers had magnesium sulfate and the results showed that there were no untoward long-term effects on the babies and their mothers. ${ }^{[1,12]}$ Therefore on the basis of high-quality evidence, WHO has recommended magnesium sulfate as the most effective, safe and low-cost drug to treat these disorders.

\section{Challenges}

However, how much of this research information has been put into practice by relevant health workers in Nigeria? This is against the backdrop that most of the doctors and midwives were trained in the use of diazepam, or 'lytic' cocktail as anticonvulsants for pre-eclampsia and eclampsia. Applying new research findings to clinical decisions is always a challenge. It has been documented that the medical profession in particular is ultimately the main constraint to change. ${ }^{[13]}$ It might be safe to assume that most obstetricians in Nigeria have accepted magnesium sulfate as the drug of choice in pre-eclampsia and eclampsia but not after series of workshops and seminars organized by the Society of Obstetrics and Gynaecology of Nigeria (SOGON) on the drug. There was also a support commentary in the specialty Journal. ${ }^{[14]}$ However, what happens on the fields is a very different ball game!

The author was surprised to find that only 3 out of 10 maternity units of tertiary hospitals that were recently visited for the purpose of postgraduate medical accreditation had magnesium sulfate on the emergency trays of their labor rooms, even though the drug was available at the hospital pharmacies. In contrast, diazepam ampoules were on the emergency trays at all the sites indicating that diazepam is the drug that is used when the emergency comes. Old habits die hard! The situation is better imagined at the secondary and primary health care centers. The implication is that the drug is still not in widespread use. Other stake holders like the general practitioners and midwives in both public and private sectors need 
the information and perhaps some persuasion. It is important to disseminate research findings to a variety of audiences, including lay readers and even journalists. The Federal Ministry of Health in collaboration with the McArthur Foundation has planned a national workshop before the end of the year to train clinicians and midwives on the use of magnesium sulfate in the management of pre-eclampsia and eclampsia. However, even at that there must be regular medical audit or monitoring to ensure standard medical practice in our health institutions otherwise the situation may not change.

Essentially, there are two standard protocols for using magnesium sulfate as anticonvulsant in preeclampsia or eclampsia. In both regimens, initiation is by the intravenous route, the difference is the route for the maintenance doses. With the Zuspan regimen, an initial intravenous bolus dose of 4 $\mathrm{g}$ is given slowly over a period of $5-10 \mathrm{~min}$ and maintenance is with $1-2 \mathrm{~g}$ hourly by intravenous infusion for $24 \mathrm{~h}$ using infusion pump. ${ }^{[15]}$ But in the absence of an infusion pump, which is what obtained in most low resource settings, the easily available gravity-fed drip sets could be used instead and then rely on the duty staff to monitor the rate of infusion. ${ }^{[16]}$ It is a task that is by no means easy in a busy maternity unit with few midwives on duty.

The second protocol is the Pritchard regimen, which is also initiated by giving $4 \mathrm{~g}$ bolus magnesium sulfate intravenously over 5-10 min and simultaneously administering $10 \mathrm{~g}$ intramuscularly ( $5 \mathrm{~g}$ each buttock). This is then followed by 5 $\mathrm{g}$ intramuscularly at 4-h intervals into alternate buttocks for 24 h. ${ }^{[17]}$ Some workers have modified this regimen by limiting the maintenance doses to 12 $\mathrm{h}$ instead of $24 \mathrm{~h}$ with good results. ${ }^{[18]}$ The Pritchard regimen is becoming more popular in low-resource settings. In Nigeria, the Federal Ministry of Health $(\mathrm{FMOH})$ recommends the Pritchard regimen ${ }^{[19]}$ and has finally put magnesium sulfate on the essential drug list.

There are concerns of magnesium sulfate toxicity especially among the older generation of physicians. Fortunately, there are clinical monitors that can be used to predict the presence or absence of toxicity. In the celebrated Magpie trial, safe monitoring was achieved without serum magnesium measurement, using simple clinical assessment of tendon reflexes, respiratory rate, and urine output. Moreover, the therapeutic index for magnesium is not as narrow as was previously thought. ${ }^{[20]}$ With any of the standard protocols, the expected serum therapeutic range of magnesium is $2.0-3.5 \mathrm{mmol} / \mathrm{l}$. A mean serum level of $2.1 \mathrm{mmol} / 1$ was reported from our unit. ${ }^{[21]}$
Nevertheless, it is advised that an ampoule of $10 \%$ calcium gluconate be made available as an antidote in any facility that uses magnesium sulfate.

In Nigeria, there is still no local production of magnesium sulfate in commercial quantity, thus availability of the drug was a major problem until recently. Courtesy of a pharmaceutical company, the drug is now available at retail outlets especially in the urban centers and cities. The next challenge was that of cost and it has been partly addressed by the pharmaceutical company. The cost of a 24-h treatment using their product (C lampex) is comparable to that of diazepam. However, there is an inherent danger in monopoly, so we need at least two companies for better coverage and healthy competition. Unfortunately, it is said that the private sector initiative alone does not work with magnesium sulfate because its production is not very profitable. The market for the drug is small and the drug is not widely used for other conditions thereby lacking the economy of scope. ${ }^{[22]}$

The drug can be made cheaper or free to patients if the government and development partners subsidize it, as it is done for anti-retroviral drugs. The United Nations Population Fund (UNFPA) and the Canadian International Development Agency (CIDA) have provided a support in that regard to states in the northwest zone and are scaling up to other states of the country. We hope that the state governments will take over the supply when these partners stop in the spirit of sustainability.

But could we have a shorter, simpler but effective regimen as a way to reduce cost? Could we also have alternative routes of administration that will enable the primary health care worker administer the drug before referring the patient? Efforts are on-going by researchers at country level to address these and other related issues (OA Ojengbede, personal communication).

Governments, developmental partners, and nongovernmental organizations concerned about women health have been urged to take all necessary measures to ensure that efforts for the prevention and treatment of eclampsia and severe pre-eclampsia are commensurate with their health care burden. ${ }^{[23]}$

\section{Conclusion}

Magnesium sulfate is the only anticonvulsant drug for which there is extensive and compelling evidence of efficacy and safety for treatment of severe preeclampsia and eclampsia. Scaling up its use in Nigeria, by addressing the barriers that limit access will significantly advance the safe motherhood 
agenda and by extension contribute to our reaching the fourth and fifth millennium development goals.

\section{References}

1. Duley L. Maternal mortality associated with hypertensive disorders of pregnancy in Africa, Asia, Latin America and the Caribbean. Br J Obstet Gynaecol 1992;99:547-53.

2. Adetoro 00. A sixteen year survey of maternal mortality associated with eclampsia in Ilorin, Nigeria. Int J Gynaecol Obstet 1989;30:117-21.

3. Ogunniyi SO, Sanusi YO, Ogunniyi FA. Eclampsia: A continuing obstetric catastrophe-the experience in Ile Ife, Nigeria. J Obstet Gynaecol 1999;19:26-9.

4. Audu LR, Ekele BA. A ten year review of maternal mortality in Sokoto, Northern Nigeria. West Afr J Med 2002;21:74-6.

5. El-Nafaty AU, Melah GS, Massa AH, Audu BM, Madziga $\mathrm{N}$. The analysis of eclamptic morbidity and mortality in Specialist Hospital Gombe, Nigeria. J Obstet Gynaecol 2004;24:142-7.

6. Ekele BA, Bello SO, Adamu AN. Clusters of eclampsia in a Nigerian teaching hospital. Int J Gynaecol Obstet 2007;96:62-6.

7. Tukur J, Umar BA, Rabi'u A. Pattern of eclampsia in a tertiary health facility in a rural town in northern Nigeria. Ann Afr Med 2007;6:164-7.

8. SOGON. Status of emergency obstetric services in six states of Nigeria - A needs assessment report. 2004.

9. EclampsiaTrial Collaborative Group. Which anticonvulsant for women with eclampsia? Evidence from the Collaborative EclampsiaTrial. Lancet 1995;345:1455-63.

10. Magpie Trial Collaborative Group. Do women with preeclampsia and their babies benefit from magnesium sulphate? The Magpie Trial: A randomized placebocontrolled trial. Lancet 2002;359:1877-90.

11. Magpie Trial Follow-up Study Collaborative Group. The MagpieTrial: A randomized trial comparing magnesium sulphate with placebo for pre-eclampsia: Outcome for children at 18 months. BJOG 2007;114:289-99.

12. Magpie Trial Follow-up Study Collaborative Group. The MagpieTrial: A randomized trial comparing magnesium sulphate with placebo for pre-eclampsia: Outcome for women at 2 years. BJOG 2007;114:300-9.

13. Garner P, Kale R, Dickson R, Dans T, Salinas R. Getting research findings into practice: Implementing research findings in developing countries. BMJ 1998;317:531-5.

14. Ekele B. Magnesium sulphate:The gold standard for the treatment of eclampsia and severe pre-eclampsia. Trop J Obstet Gynaecol 2006;23:1-2.

15. Zuspan FP. Problems encountered in the treatment of pregnancy induced hypertension. Am J Obstet Gynecol 1978;131:591-7.

16. Adewole IF, Oladokun A, Okewole AI, Omigbodun AO, Afolabi A, Ekele B, et al. Magnesium sulphate for treatment of eclampsia: The Nigerian experience. Afr J Med Med Sci 2000;29:239-41.

17. Pritchard JA, Cunningham FG, Pritchard SA. The Parkland Memorial Hospital protocol for treatment of eclampsia: Evaluation of 245 cases. Am J Obstet Gynecol 1984;148:951-63.

18. Ekele BA, Ahmed Y. Magnesium sulfate regimens for eclampsia. Int J Gynaecol Obstet 2004;87:149-50.

19. $\mathrm{FMOH}$. National Clinical Service Protocol for Obstetrics and Neonatal Care, 2006.

20. LuJF, Nightingale CH. Magnesium sulphate in eclampsia and pre-eclampsia: Pharmacokinetic principles. Clin Pharmacokinet 2000;38:305-14.

21. Ekele BA, Badung SL. Is serum magnesium estimate necessary in patients with eclampsia on magnesium sulphate? Afr J Reprod Health 2005;9:128-32.

22. Sevene E, Lewin S, Mariano A, Woelk G, Oxman AD, Matinhure $S$, et al. System and market failures: The unavailability of magnesium sulphate for the treatment of eclampsia and pre-eclampsia in Mozambique and Zimbabwe. BMJ 2005;331:765-9.

23. Langer A, Villar J, Tell K, Kim T, Kennedy S. Reducing eclampsia related deaths: A clarion call to action. Lancet 2008;371:705-6. 